\title{
Antisymmetric rank-2 tensor unparticle physics
}

\author{
Tae-il Hur ${ }^{a b}$, P. $\mathrm{Ko}^{b}$ and Xiao-Hong $\mathrm{Wu}^{b}$ \\ a Department of Physics, KAIST, Daejeon 305-701, Korea \\ ${ }^{b}$ School of Physics, Korea Institute for Advanced Study, 207-43, \\ Cheongryangri 2-dong, Dongdaemun-gu, Seoul 130-722, Korea
}

\begin{abstract}
We present the phenomenology of antisymmetric rank-2 tensor unparticle operator $\mathcal{O}_{\mathcal{U}, \mathcal{A}}^{\mu \nu}$ with scaling dimension $d_{\mathcal{U}}$. We consider the physical effects of operator $O_{\mathcal{U}, \mathcal{A}}^{\mu \nu}$ in $Z^{0}$ boson invisible decays $Z^{0} \rightarrow \mathcal{U}, Z^{0} \rightarrow b \bar{b}$ channel, the electroweak precision observable $S$ parameter, and the muon anomalous magnetic dipole moment. The $Z^{0}$ boson invisible decay gives a very stringent constraint in the $\left(\Lambda_{\mathcal{U}}, M_{\mathcal{U}}\right)$ plane, and only small $r \equiv \Lambda_{\mathcal{U}} / M_{\mathcal{U}} \lesssim 0.1$ is favored, when $\Lambda_{\mathcal{U}}$ is order of several $100 \mathrm{GeV}$. When the phenomenological parameter $\mu$, which parameterizes the scale invariance breaking, goes to 0 , the $S$ parameter and the muon $(g-2)$ diverge for $1<d_{\mathcal{U}}<2$, while for non-zero $\mu$, there will be constraints on $\left(\Lambda_{\mathcal{U}}, M_{\mathcal{U}}\right)$ which are more stringent than those obtained from collider experiments.
\end{abstract}




\section{INTRODUCTION}

Recently Georgi proposed an interesting possibility that there might be a hidden sector with operators $O_{U V}$ 's that flows into scale invariant theory at low energy scale $\Lambda_{\mathcal{U}}$ with operators $O_{\mathcal{U}}$ 's. The hidden sector operators $O_{U V}$ 's in the UV theory can interact with the SM sector by nonrenormalizable interactions that are generated at some scale $M_{\mathcal{U}}$, which match to the scale invariant interaction with the Standard Model (SM) sector below $\Lambda_{\mathcal{U}}$ [1, 2]. Schematically, one has the following picture:

$$
C_{n} \frac{\mathcal{O}_{S M} \mathcal{O}_{U V}}{M_{U}^{d_{U V}+n-4}} \longrightarrow C_{n}^{i} \frac{\Lambda_{\mathcal{U}}^{d_{U V}-d_{\mathcal{U}}}}{M_{U}^{d_{U V}+n-4}} \mathcal{O}_{S M, i}^{n} \mathcal{O}_{\mathcal{U}} \equiv \frac{C_{n}^{i}}{\Lambda_{n}^{d_{\mathcal{U}}+n-4}} \mathcal{O}_{S M, i}^{n} \mathcal{O}_{\mathcal{U}}
$$

where $d_{U V}, d_{\mathcal{U}}$ and $n$ are the scaling dimensions of the UV operator in the Banks-Zaks (BZ) sector [3], the unparticle operator $O_{\mathcal{U}}$, and the SM operator of a type $i, \mathcal{O}_{S M, i}^{n}$. Triggered by this intriguing suggestion, a lot of phenomenological analysis have been done for both low and high energy processes involving unparticle operators [4, 5, 6, 7, 8, 9, 10, 11, 12, 13, 14, $15,16,17,18,19,20,21,22,23,24,25,26,27,28,29,30,31,32,33,34,35,36,37,38,39$, 40, 41, 42, 43, 44, 45, 46, 47, 48, 49, 50, 51, 52, 53, 54, 55, 56, 57. . So far, most works have considered scalar and vector unparticle operators, and a few works on the rank-2 symmetric tensors, especially its modification of the Newtonian gravity potential.

In this letter, we study the antisymmetric rank-2 tensor unparticle operator, $\mathcal{O}_{\mathcal{U}, \mathcal{A}}^{\mu \nu}$ : its spectral representation, propagator and phenomenological implications. This operator has a unique property that it can have coupling with the dimension-2 SM operator $B_{\mu \nu}$, the field strength tensor of $U(1)_{Y}$ gauge boson. Therefore it can contribute to the invisible decay width of $Z^{0}$ boson. Also it can modify $Z \rightarrow b \bar{b}$, the Peskin-Takeuchi $S$ parameter, and the muon $(g-2)$, when we simultaneously consider its interactions with other SM operators. In the following, we consider these observables in the presence of antisymmetric rank-2 tensor unparticle operator.

Before closing this section, we would like to define the conventions and the normalizations of interactions between the unparticle operators and the SM. In most papers on unparticle phenomenology, the scale $\Lambda_{n}$ in Eq. (1) is widely used, and bounds on them are derived assuming the coupling $C_{n}^{i}=O(1)$. However these scales $\Lambda_{n}$ 's are derived from the first line of Eq. (1), and it depends on the dimension of the SM operator that couples to, and in general $\Lambda_{3} \neq \Lambda_{4}$. Scales $\Lambda_{n}$ 's can be expressed in terms of fundamental scale $M_{U}$ and $\Lambda_{\mathcal{U}}$, and the scaling dimensions $d_{\mathrm{UV}}, d_{\mathcal{U}}$ and $n$. The expressions for $\Lambda_{i=2,3,4}$ can be found in Ref. [25]:

$$
\Lambda_{2}=r^{\frac{d_{\mathrm{UV}}-2}{2-d_{\mathcal{U}}}} \Lambda_{\mathcal{U}}, \quad \Lambda_{3}=\left(\frac{1}{r}\right)^{\frac{d_{\mathrm{UV}}-1}{d_{\mathcal{U}}-1}} \Lambda_{\mathcal{U}}, \quad \Lambda_{4}=\left(\frac{1}{r}\right)^{\frac{d_{\mathrm{UV}}}{d_{\mathcal{U}}}} \Lambda_{\mathcal{U}},
$$

where $r \equiv \Lambda_{\mathcal{U}} / M_{\mathcal{U}}$ is less or equal to 1 by definition. For $1<d_{\mathcal{U}}<2<d_{U V}$, one has

$$
\Lambda_{2}<M_{U}<\Lambda_{4}<\Lambda_{3}
$$

In this analysis, we will follow the conventions of Ref. [25] for the scales $\Lambda_{n}$ 's and the couplings $C_{n}^{i}$ 's and study various observables that could be affected by the antisymmetric rank-2 tensor unparticle operator $O_{\mathcal{U}, \mathcal{A}}^{\mu \nu}$. Then the bounds on the scales $\Lambda_{n}$ 's will be cast into those on $\left(\Lambda_{\mathcal{U}}, M_{\mathcal{U}}\right)$ for $d_{U V}=3$. This will facilitate the comparison with the existing bounds in much clearer way. 


\section{TWO-POINT FUNCTION AND PROPAGATOR}

The antisymmetric tensor $O_{\mathcal{U}, A}^{\mu \nu}=-O_{\mathcal{U}, A}^{\nu \mu}$ can be decomposed into magnetic and electric vectors, with the index $\mu \nu=i j, i, j=1,2,3$ for magnetic components, and $\mu \nu=0 i$ for electric components [58]. Using the scale symmetry, the two-point function for $O_{\mathcal{U}, A}^{\mu \nu}$ can be written as

$$
\left\langle 0\left|O_{\mathcal{U}, A}^{\mu \nu}(x) O_{\mathcal{U}, A}^{\rho \sigma}(0)\right| 0\right\rangle=\int \frac{d^{4} p}{(2 \pi)^{4}} e^{-i p x} A_{d_{\mathcal{U}}} \theta\left(p^{0}\right) \theta\left(p^{2}\right) \Pi^{\mu \nu \rho \sigma}\left(p^{2}\right)^{d_{\mathcal{U}}-2},
$$

where the projection operator $\Pi^{\mu \nu \rho \sigma}$ is defined as follows [58]:

$$
\Pi^{\mu \nu \rho \sigma}=\frac{1}{2}\left(P^{\mu \rho} P^{\nu \sigma}-P^{\mu \sigma} P^{\nu \rho}\right)
$$

for magnetic component, and

$$
\Pi^{\mu \nu \rho \sigma}=\frac{1}{2}\left(P^{\mu \rho} \omega^{\nu \sigma}-P^{\mu \sigma} \omega^{\nu \rho}-P^{\nu \rho} \omega^{\mu \sigma}+P^{\nu \sigma} \omega^{\mu \rho}\right)
$$

for electric part, with

$$
P^{\mu \nu}=g^{\mu \nu}-p^{\mu} p^{\nu} p^{-2}, \quad \omega^{\mu \nu}=p^{\mu} p^{\nu} p^{-2} .
$$

We use the same normalization for the overall factor $A_{d_{\mathcal{U}}}$ as Georgi [1, 2] :

$$
A_{d_{\mathcal{U}}}=\frac{16 \pi^{5 / 2}}{(2 \pi)^{2 d}} \frac{\Gamma(d+1 / 2)}{\Gamma(d-1) \Gamma(2 d)} .
$$

It is straightforward to derive the propagator using the above expression for the two-point function:

$$
\left\langle 0\left|T\left(O_{\mathcal{U}, A}^{\mu \nu}(x) O_{\mathcal{U}, A}^{\rho \sigma}(0)\right)\right| 0\right\rangle=i \frac{A_{d_{\mathcal{U}}}}{2} \int \frac{d^{4} p}{(2 \pi)^{4}} e^{-i p x} \frac{\Pi^{\mu \nu \rho \sigma}}{\sin \left(d_{\mathcal{U}} \pi\right)}\left(-p^{2}-i \epsilon\right)^{d_{\mathcal{U}}-2}
$$

These two equations (3) and (41) are one of the main results of this paper, and necessary ingredient in order that we calculate physical observables in the following.

In addition to $\mathcal{O}_{\mathcal{U}, \mathcal{A}}^{\mu \nu}$, there is one more unparticle operator that can couple to the dimension-2 SM operator $H^{\dagger} H[14]$ :

$$
\frac{c_{2}}{\Lambda_{2}^{d_{U}-2}} \mathcal{O}_{S} H^{\dagger} H
$$

After the EWSB, this operator will generate a mass scale $\mu$, and thus induces conformal symmetry breaking in the unparticle sector. Bander et al. [25] argue that

$$
\left(\frac{\mu}{\Lambda_{4}}\right) \lesssim\left(10^{-3}\right)^{1 / d_{\mathcal{U}}}
$$

Following Ref. [14], we parameterize this conformal symmetry breaking by phenomenological effective mass scale $\mu$, and modify the two-point function and propagator as follows:

$$
\begin{aligned}
\left\langle 0\left|O_{\mathcal{U}, A}^{\mu \nu}(x) O_{\mathcal{U}, A}^{\rho \sigma}(0)\right| 0\right\rangle & =\int \frac{d^{4} p}{(2 \pi)^{4}} e^{-i p x} A_{d_{\mathcal{U}}} \theta\left(p^{0}\right) \theta\left(p^{2}-\mu^{2}\right) \Pi^{\mu \nu \rho \sigma}\left(p^{2}-\mu^{2}\right)^{d_{\mathcal{U}}-2} \\
\left\langle 0\left|T\left(O_{\mathcal{U}, A}^{\mu \nu}(x) O_{\mathcal{U}, A}^{\rho \sigma}(0)\right)\right| 0\right\rangle & =i \frac{A_{d_{\mathcal{U}}}}{2} \int \frac{d^{4} p}{(2 \pi)^{4}} e^{-i p x} \frac{\Pi^{\mu \nu \rho \sigma}}{\sin \left(d_{\mathcal{U}} \pi\right)}\left[-\left(p^{2}-\mu^{2}\right)-i \epsilon\right]^{d_{\mathcal{U}}-2}
\end{aligned}
$$


In the following, we find that the contributions of an antisymmetric rank-2 tensor unparticle operator to the $S$ parameter and the muon $(g-2)$ are proportional to $\left(\mu^{2}\right)^{d_{\mathcal{U}}-2}$, so that they are divergent for $\mu=0$ and $1<d_{\mathcal{U}}<2$. Therefore we have to keep the nonzero $\mu$ scale for some observables considered in this work.

\section{PHENOMENOLOGY}

In this section, we consider the physical effects of antisymmetric unparticle operator $O_{\mathcal{U}, A}^{\mu \nu}$ of scaling dimension $d_{\mathcal{U}}$ on the invisible decay width of $Z^{0}$ boson $(Z \rightarrow \mathcal{U}), R_{b}$ and $A_{\mathrm{FB}}^{b}$ in the $Z \rightarrow b \bar{b}$ decay channel, an electroweak precision observable $S$ parameter, and the muon anomalous magnetic dipole moment. The relevant interaction terms involving the antisymmetric rank-2 tensor unparticle operator $O_{\mathcal{U}, A}^{\mu \nu}$ can be written as

$$
\begin{aligned}
\mathcal{L}_{\text {int }} & =\lambda_{b} \frac{g^{\prime}}{\Lambda_{2}^{d_{\mathcal{U}}-2}} B_{\mu \nu} O_{\mathcal{U}, A}^{\mu \nu}+\widetilde{\lambda_{b}} \frac{g^{\prime}}{\Lambda_{2}^{d_{\mathcal{U}}-2}} \widetilde{B_{\mu \nu}} O_{\mathcal{U}, A}^{\mu \nu} \\
& +\lambda_{w} \frac{g}{\Lambda_{4}^{d_{\mathcal{U}}}}\left(H^{\dagger} \tau^{a} H\right) W_{\mu \nu}^{a} O_{\mathcal{U}, A}^{\mu \nu}+\lambda_{f} \frac{y_{f}}{\Lambda_{4}^{d_{\mathcal{U}}}} \bar{f}_{L} H \sigma_{\mu \nu} f_{R} O_{\mathcal{U}, A}^{\mu \nu} .
\end{aligned}
$$

Here the field strength tensor $B_{\mu \nu}$ of the $U(1)_{Y}$ gauge boson and its dual $\widetilde{B_{\mu \nu}}$ are gauge invariant dimension-2 operators, and the other two SM operators are of dimension 4 . We included the gauge couplings for gauge fields, and the Yukawa couplings for Higgs-fermion couplings, and assumed that the couplings $\lambda_{i}$ 's are all order $O(1)$. This is in accord with Ref. [25] and we can compare directly our results on $\left(\Lambda_{\mathcal{U}}, M_{\mathcal{U}}\right)$ with their results on scalar and vector unparticle operators derived from LEP/SLC.

Note that the first two terms are unique to the antisymmetric rank-2 tensor unparticle operator $O_{\mathcal{U}, A}^{\mu \nu}$ we consider in this work. As mentioned in the previous section, there are only two operators $O_{\mathcal{U}, A}^{\mu \nu}$ and the scalar unparticle operator $\mathcal{O}_{\mathcal{U}, \mathcal{S}}$ that can couple to the dimension-2 SM operators, which are $B_{\mu \nu}$ and $H^{\dagger} H$, respectively.

\section{A. $Z^{0} \rightarrow \mathcal{U}$}

In the presence of antisymmetric rank-2 tensor unparticle operator, $Z^{0}$ boson can decay to an invisible unparticle, $Z^{0} \rightarrow \mathcal{U}$ through (i) the 1 st term alone and (ii) the second order effects involving the 1st and the 3rd terms in Eq. (7). The 2nd term of Eq.(7) does not give a nonvanishing result due to the Levi-Civita tensor. Using the results obtained in Sec. II, we can calculate the $Z^{0} \rightarrow \mathcal{U}$ decay width easily:

$$
\Gamma\left(Z^{0} \rightarrow U\right)=2 g^{\prime 2} s_{w}^{2} m_{Z} A_{d_{\mathcal{U}}} \lambda_{b}^{2}\left(\frac{m_{Z}^{2}-\mu^{2}}{\Lambda_{2}^{2}}\right)^{d_{\mathcal{U}}-2}\left(1+\frac{c_{w}}{4 s_{w}} \frac{\lambda_{w}}{\lambda_{b}} \frac{v^{2}}{\Lambda_{4}^{2}} \frac{\Lambda_{2}^{d_{\mathcal{U}}}-2}{\Lambda_{4}^{d_{\mathcal{U}}-2}}\right)^{2} \theta\left(m_{Z}^{2}-\mu^{2}\right)
$$

where $s_{w} \equiv \sin ^{2} \theta_{W}$ and $c_{w} \equiv \cos \theta_{W}$. We note that in the $Z$ rest frame, only the electric component of antisymmetric tensor contributes to the invisible $Z \rightarrow \mathcal{U}$ decay. The contributions of a vector unparticle operator $\partial^{\mu} O_{V}^{\nu}$ coupled to dimension-2 SM operator $B_{\mu \nu}$ have already been considered in ref. [29]. However the operator we considered here, the term with dimension-2 SM operator in Eq. (7) gives the dominant contribution. 
The $Z^{0}$ boson properties have been well studied, and its invisible decay width is quite consistent with the SM predictions with three light neutrinos:

$$
\Gamma_{\exp }^{\text {invis }}\left(Z^{0}\right)=(499.0 \pm 1.5) \mathrm{MeV}, \text { vs. } \Gamma_{\mathrm{SM}}\left(Z^{0} \rightarrow \nu \bar{\nu}\right)=(501.65 \pm 0.11) \mathrm{MeV}
$$

This leaves little room to an additional invisible decay width of $Z^{0}$ boson, for example, into the unparticle sector. We assume that the room for the invisible $Z^{0} \rightarrow \mathcal{U}$ decay is the uncertainty of experimental uncertainty in $\Gamma^{\text {invis }}$, namely $1.5 \mathrm{MeV}$.

In the numerical analyses of $Z^{0} \rightarrow \mathcal{U}$, we fix the dimensionless coupling to $1, d_{\mathrm{UV}}=3$ for $d_{\mathcal{U}}=1.1,1.5,1.9$, and choose two different $\mu=0$ and $\mu=85 \mathrm{GeV}$. We note that the variation of $\mu$ parameter with $\mu<m_{Z}$ does not affect the results very much, as $\mu$ enters Eq. (8) through the combination of $\left(m_{Z}^{2}-\mu^{2}\right)^{d_{\mathcal{U}}-2}$. The contour plots for $\Gamma\left(Z^{0} \rightarrow \mathcal{U}\right)$ in the $\left(\Lambda_{\mathcal{U}}, M_{\mathcal{U}}\right)$ plane is shown in Fig. 1, The dash lines in the figure correspond to the contribution from the first term in Eq. (7) only, which is the contribution in Eq. (86) without the second term $\frac{c_{w}}{4 s_{w}} \ldots$ in the squared bracket. We find that the contribution from second term $\frac{c_{w}}{4 s_{w}} \ldots$ is not small when $\Lambda_{\mathcal{U}}$ is below several tens of $\mathrm{GeV}$, while for larger $\Lambda_{\mathcal{U}}$, the contribution is dominated by the first term, as we can see that the dash and solid line overlap. This is because the second term $\frac{c_{w}}{4 s_{w}} \frac{\lambda_{w}}{\lambda_{b}} \frac{v^{2}}{\Lambda_{4}^{2}} \frac{\Lambda_{2}^{d_{\mathcal{U}}-2}}{\Lambda_{4}^{d_{\mathcal{U}}-2}}=\frac{c_{w}}{4 s_{w}} \frac{\lambda_{w}}{\lambda_{b}} \frac{v^{2}}{\Lambda_{\mathcal{U}}^{2}} r^{2} \sim \frac{v^{2}}{\Lambda_{\mathcal{U}}^{2}}$ with $r=\frac{\Lambda_{\mathcal{U}}}{M_{\mathcal{U}}} \leq 1$, when we replace $\Lambda_{2,4}$ with $M_{\mathcal{U}}$ and $\Lambda_{\mathcal{U}}$. That factor is suppressed when $\Lambda_{\mathcal{U}}$ is larger than the electroweak scale $v$. Hence, the contribution from the third term of Eq. (7) is suppressed compared with the one from the first term. We also change the sign of dimensionless parameter $\lambda_{w}$ to -1 in case (a) and (c) to show the contribution of the second term of Eq. (8), because $\lambda_{w}$ only enter the second term in the formula. For larger $\Lambda_{\mathcal{U}}$ of order $100 \mathrm{GeV}$, the second term is suppressed, the curve in case (a) and (c) is similar to (b) and (d) in the large $\Lambda_{\mathcal{U}}$ region. We have similar results in the discussion of other physical quantities later on. We find that the constraint from the invisible decay of $Z$ is very stringent, only small $r=\Lambda_{\mathcal{U}} / M_{\mathcal{U}} \lesssim 0.1$ is favored. We note that $\Gamma\left(Z^{0} \rightarrow \mathcal{U}\right)=0$ if $\mu \geq m_{Z}$, due to the $\theta$ function in Eq. (8) , and there would be no constraint from the invisible $\bar{Z}^{0}$ decay width on the unparticle physics. Comparing Fig. 1 with the plots in Ref. [25], we can conclude that the constraint from the invisible $Z^{0}$ decay width is much more stringent than those from the effects of scalar and vector unparticle operators at LEP/SLC experiments as long as $\mu<m_{Z}$.

\section{B. $Z^{0} \rightarrow \bar{b} b$}

The decay $Z^{0} \rightarrow b \bar{b}$ is an important process not only within the SM, but also in many models beyond the SM, since any new physics scenarios which deals the 3rd generation quarks differently from the light two families can affect this decay, and thus is strongly constrained by electroweak precision data. In the model independent effective theory approach, this channel has been discussed in [61] including the magnetic operators. In the framework of unparticle physics, vector unparticle contribution to $Z^{0} \rightarrow \bar{b} b$ has been investigated in ref. [47].

The antisymmetric rank-2 tensor unparticle operator $O_{\mathcal{U}, A}^{\mu \nu}$ can contribute to the magnetic operator in the decay $Z^{0} \rightarrow b \bar{b}$ through $\lambda_{b}$ and $\lambda_{f}$ terms in Eq. (7). We first calculate the coefficient $g_{T}^{b}$ of the magnetic operator defined in [61], and discuss the physical quantities $A_{\mathrm{FB}}^{b}$ and $R_{b}$.

$$
g_{T}^{b}=s_{w}^{2} \lambda_{b} \lambda_{f} \frac{A_{d_{\mathcal{U}}} e^{-i\left(d_{\mathcal{U}}-2\right) \pi}}{2 \sin \left(d_{\mathcal{U}} \pi\right)} \frac{m_{b} m_{z}\left(m_{z}^{2}-\mu^{2}\right)^{d_{\mathcal{U}}-2}}{\Lambda_{2}^{d_{\mathcal{U}}-2} \Lambda_{4}^{d_{\mathcal{U}}}}\left(1+\frac{c_{w}}{4 s_{w}} \frac{g}{g^{\prime}} \frac{\lambda_{w}}{\lambda_{b}} \frac{v^{2}}{\Lambda_{4}^{2}} \frac{\Lambda_{2}^{d_{\mathcal{U}}-2}}{\Lambda_{4}^{d_{\mathcal{U}}-2}}\right)
$$



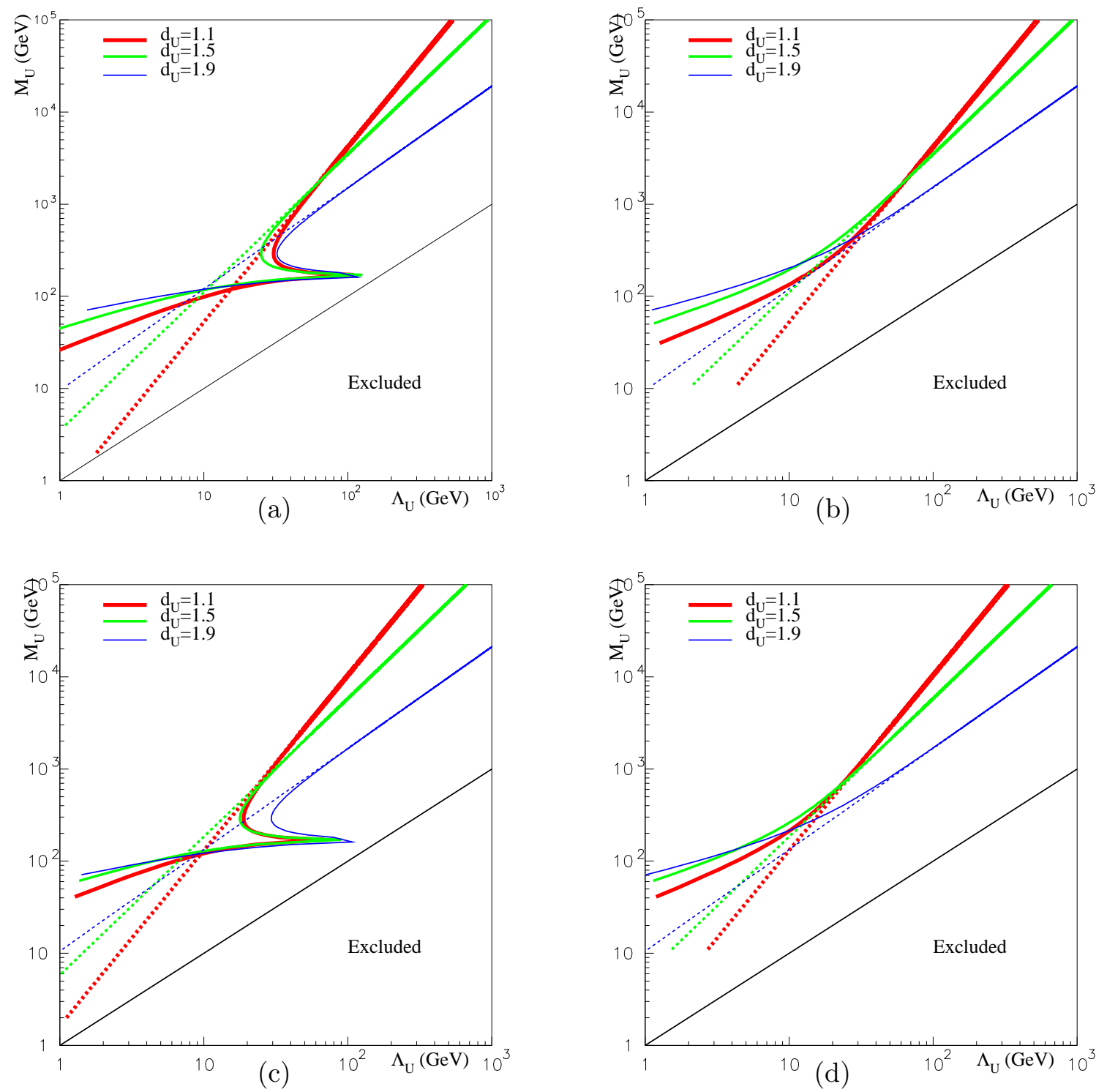

FIG. 1: The contour plot of $\Gamma(Z \rightarrow U)$ with $\Gamma(Z \rightarrow U)=1.5 \mathrm{MeV}$ in $\Lambda_{\mathcal{U}}$ and $M_{\mathcal{U}}$ plane for $d_{\mathcal{U}}=1.1$ (red), $d_{\mathcal{U}}=1.5$ (green), and $d_{\mathcal{U}}=1.9$ (blue). (a) and (b) correspond to $\mu=0$, (c) and (d) correspond to $\mu=85 \mathrm{GeV}$. $\lambda_{b}=-\lambda_{w}=1$ in case (a) and (c), $\lambda_{b}=\lambda_{w}=1$ in case (b) and (d). In the region above the corresponding curve for different $d_{\mathcal{U}}, \Gamma(Z \rightarrow U)$ is smaller than $1.5 \mathrm{MeV}$. The region below $\Lambda_{\mathcal{U}}=M_{\mathcal{U}}$ (black) is excluded.

Experimentally, both $A_{\mathrm{FB}}^{b}$ and $R_{b}$ have been determined precisely, $A_{\mathrm{FB}}^{b}=0.923 \pm 0.020$, $R_{b}=0.21629 \pm 0.00066$. We fix the dimensionless parameters to $1, d_{\mathrm{UV}}=3$ for $d_{\mathcal{U}}=1.1$ $1.5,1.9$, and choose $\mu=0$. The contour plot of $A_{\mathrm{FB}}^{b}$ and $R_{b}$ is presented in Fig. 2. Most of the region above the $\Lambda_{\mathcal{U}}=M_{\mathcal{U}}$ (black) line is allowed by the $1 \sigma$ bound of both $A_{\mathrm{FB}}^{b}$ and $R_{b}$. In that region, the unparticle effect is quit small and can be neglected. We note that the variation of $\mu$ parameter does not affect the results very much, as $\mu$ enters Eq. (9) through the combination of $\left(m_{Z}^{2}-\mu^{2}\right)^{d_{\mathcal{U}}-2}$. 

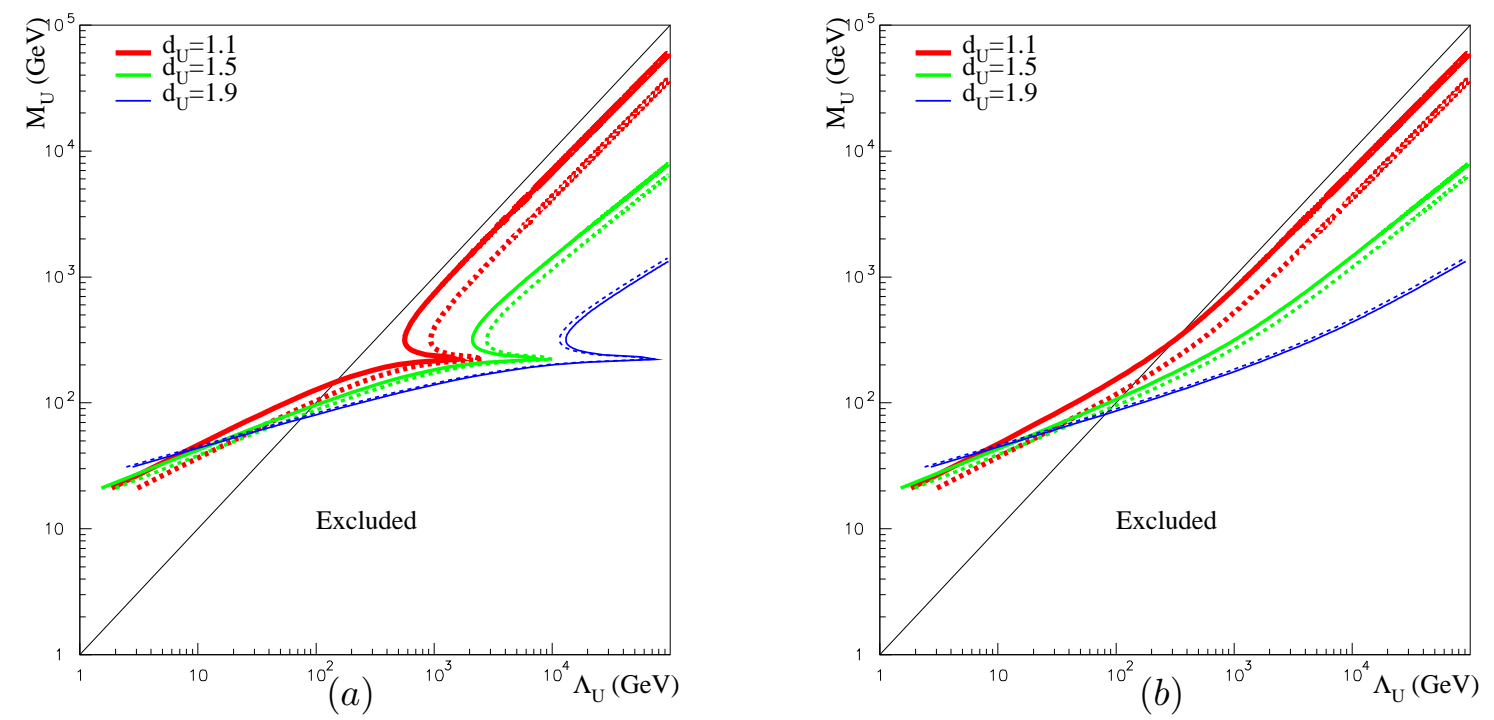

FIG. 2: The contour plot of $A_{\mathrm{FB}}^{b}$ (dash) and $R_{b}$ (solid) within $1 \sigma$ bound in $\Lambda_{\mathcal{U}}$ and $M_{\mathcal{U}}$ plane for $d_{\mathcal{U}}=1.1$ (red), $d_{\mathcal{U}}=1.5$ (green), and $d_{\mathcal{U}}=1.9$ (blue) with $\mu=0 . \quad \lambda_{f}=\lambda_{b}=-\lambda_{w}=1$ in case (a), $\lambda_{f}=\lambda_{b}=\lambda_{w}=1$ in case (b). The region above the corresponding curve for different $d_{\mathcal{U}}$ is allowed with $1 \sigma$ bound.

\section{C. $S$ parameter}

The Peskin-Takeuchi parameters, $S, T$ and $U$, have been introduced in order to constrain new physics contributions to the gauge boson self energy. The unparticle $O_{\mathcal{U}, A}^{\mu \nu}$ exchange through $\lambda_{b}$ and $\lambda_{w}$ terms can induce the dimension- 6 operator $\left(H^{\dagger} \tau^{a} H\right) W_{\mu \nu}^{a} B^{\mu \nu}$, which is directly related with the $S$ parameter:

$$
S=2 \frac{c_{w}}{s_{w}} g^{2} g^{\prime 2} \lambda_{b} \lambda_{w} \frac{A_{d_{\mathcal{U}}}}{2 \sin \left(d_{\mathcal{U}} \pi\right)} \frac{v^{2}\left(\mu^{2}\right)^{d_{\mathcal{U}}-2}}{\Lambda_{2}^{d_{\mathcal{U}}-2} \Lambda_{4}^{d_{\mathcal{U}}}} .
$$

Let us note that the $S$ parameter is divergent if $\mu^{2}=0$ for $1<d_{\mathcal{U}}<2$. This problem disappears if $d_{\mathcal{U}}>2$, but we do not consider this possibility here.

The overall $S$ is the sum of the unparticle and the SM contributions, where we assume a light SM Higgs $m_{H}=120 \mathrm{GeV}$, and $S_{\mathrm{SM}}=-0.22$. From the electroweak precision data, the $\mathrm{S}$ is determined as $S=-0.13 \pm 0.10$ [59]. In our numerical results, we choose dimensionless couplings to $1, d_{\mathrm{UV}}=3$ for $d_{\mathcal{U}}=1.1,1.5,1.9$, and choose $\mu=1 \mathrm{GeV}$ and $\mu=m_{Z}$. We plot the contour diagram of the $S$ parameter with $1 \sigma$ bound in $\left(\Lambda_{\mathcal{U}}, M_{\mathcal{U}}\right)$ plane for different $d_{\mathcal{U}}$ 's specified in the caption in Fig. 3 (a), (c) with different sign of $\lambda_{b}$ and $\lambda_{w}$ and Fig. 3 (b), (d) with the same signs. In Fig. 3 (a), (c), the contribution from unparticle is positive, and the curve in the plot corresponds to $1 \sigma$ upper bound $S=-0.03$, while the curve in Fig.(3b) corresponds to $1 \sigma$ lower bound $S=-0.23$, and unparticle contribution is negative, and hence constructive with the SM contribution. The region above the curve is allowed by the $1 \sigma$ bound of the $S$ parameter. 

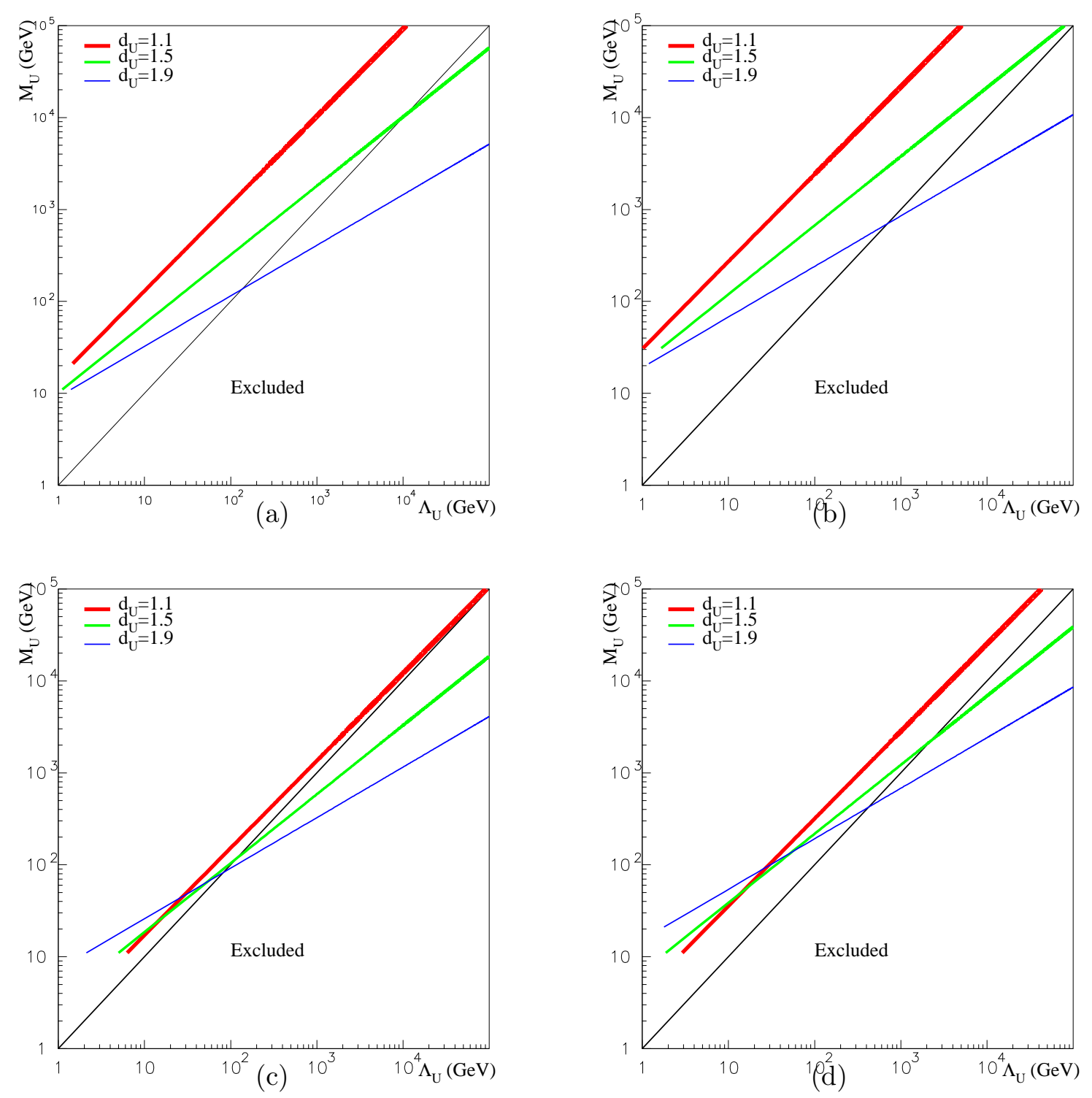

FIG. 3: The contour plot of the $S$ parameter within $1 \sigma$ bound in $\Lambda_{\mathcal{U}}$ and $M_{\mathcal{U}}$ plane for $d_{\mathcal{U}}=1.1$ (red), $d_{\mathcal{U}}=1.5$ (green), and $d_{\mathcal{U}}=1.9$ (blue) with $\mu=1 \mathrm{GeV}$ in (a), (b) and $\mu=m_{Z}$ in (c), (d). $\lambda_{b}=-\lambda_{w}=1$ in case (a) and (c), $\lambda_{b}=\lambda_{w}=1$ in case (b) and (d). The region above the corresponding curve for different $d_{\mathcal{U}}$ is allowed with $1 \sigma$ bound.

\section{Muon anomalous magnetic dipole moment $(g-2)_{\mu}$}

The muon anomalous magnetic dipole moment $(g-2)$ is a testing ground of the SM at quantum levels, and also a sensitive probe to new physics scenarios at EW scales. At present, the experimental data and the SM prediction have 3.4 $\sigma$ deviation [60],

$$
a_{\mu}^{\exp }-a_{\mu}^{\mathrm{SM}}=(29.5 \pm 8.8) \times 10^{-10} .
$$

The antisymmetric rank-2 tensor unparticle operator can contribute to the muon $(g-2)_{\mu}$ 
via both $\lambda_{b}-\lambda_{f}$ and $\lambda_{f}-\lambda_{w}$ interactions. We can derive the contribution $a_{\mu} \equiv \frac{1}{2}(g-2)_{\mu}$ as

$$
a_{\mu}^{\mathcal{U}}=\lambda_{b} \lambda_{f} \frac{A_{d_{\mathcal{U}}}}{2 \sin \left(d_{\mathcal{U}} \pi\right)} \frac{m_{\mu}^{2}\left(\mu^{2}\right)^{d_{\mathcal{U}}-2}}{\Lambda_{2}^{d_{\mathcal{U}}-2} \Lambda_{4}^{d_{\mathcal{U}}}}\left(1-\frac{s_{w}}{4 c_{w}} \frac{g}{g^{\prime}} \frac{\lambda_{w}}{\lambda_{b}} \frac{v^{2}}{\Lambda_{4}^{2}} \frac{\Lambda_{2}^{d_{\mathcal{U}}-2}}{\Lambda_{4}^{d_{\mathcal{U}}-2}}\right) .
$$

Note that this is divergent for $\mu=0$ and $1<d_{\mathcal{U}}<2$, and we choose a nonzero $\mu$ in the numerical analysis: $\mu=1 \mathrm{GeV}$ and $\mu=m_{Z}$.

In the numerical calculation, we fix the dimensionless couplings to $1 d_{\mathrm{UV}}=3$ for $d_{\mathcal{U}}=1.1$, 1.5, 1.9. There could be additional contributions to the muon $(g-2)$ from other unparticle operators. Therefore, instead of fitting the muon $(g-2)$ by the antisymmetric rank-2 tensor unparticle operator, we assume its contribution is smaller than $\sim 10^{-9}$. In Fig. 4, we show the contour plots of unparticle induced $a_{\mu}$ with $a_{\mu}^{\mathcal{U}}=10^{-9}$ in the $\left(\Lambda_{\mathcal{U}}, M_{\mathcal{U}}\right)$ plane. We find that the contribution from unparticle can be as large as $10^{-9}$ for $d_{\mathcal{U}}=1.1,1.5,1.9$ in the region below the curves, which are disfavored accordingly. Note that the constraint from the muon $(g-2)$ is stronger than those obtained from LEP/SLC data [25].

\section{CONCLUSIONS}

In this paper, we concentrated on the antisymmetric rank-2 tensor unparticle operator $\mathcal{O}_{\mathcal{U}, \mathcal{A}}^{\mu \nu}$ with scaling dimension $1<d_{\mathcal{U}}<2$. This operator has a unique property that it can mix with the $B_{\mu \nu}$ operator of the SM sector, and can modify the properties of $Z^{0}$ bosons. We first derived the two-point function and the propagator for the antisymmetric rank-2 tensor unparticle operator, studied their effects on $Z^{0}$ boson properties, the invisible $Z^{0}$ decay width $Z^{0} \rightarrow \mathcal{U}, Z^{0} \rightarrow b \bar{b}$, the $S$ parameter and the muon $(g-2)_{\mu}$. We find that the last two observables are divergent for $1<d_{\mathcal{U}}<2$. Therefore we had to introduce a phenomenological parameter $\mu$, a low energy scale where scale symmetry is broken, in order to make $S$ parameter and the muon $(g-2)$ finite. The most stringent bounds on the fundamental scales $\Lambda_{\mathcal{U}}$ and $M_{\mathcal{U}}$ come from the invisible decay width $Z^{0} \rightarrow \mathcal{U}$ for $\mu<m_{Z}$. We find $r=\Lambda_{\mathcal{U}} / M_{\mathcal{U}} \lesssim 0.1$ is favored, which is more stringent than the bounds from LEP/SLC [25]. Furthermore the contributions of $\mathcal{O}_{\mathcal{U}, \mathcal{A}}^{\mu \nu}$ to the $S$ parameter and the muon $(g-2)$ are proportional to $\left(\mu^{2}\right)^{d_{\mathcal{U}}-2}$, which is divergent for $\mu^{2}=0$ for $1<d_{\mathcal{U}}<2$. Therefore we need nonzero $\mu^{2}$ for $1<d_{\mathcal{U}}<2$. For $\mu \neq 0$ (especially for $\mu \geq m_{Z}$ ), there would be no constraint from the invisible $Z^{0}$ decay width or from astrophysical processes involving unparticle emissions from stars and supernovae. Still the constraint from the muon $(g-2)_{\mu}$ is quite significant for $\mu \neq 0$.

\section{Acknowledgments}

This work is supported in part by KOSEF through CHEP at Kyungpook National University.

[1] H. Georgi, Phys. Rev. Lett. 98, 221601 (2007) arXiv:hep-ph/0703260.

[2] H. Georgi, arXiv:0704.2457 [hep-ph].

[3] T. Banks and A. Zaks, Nucl. Phys. B 196, 189 (1982). 

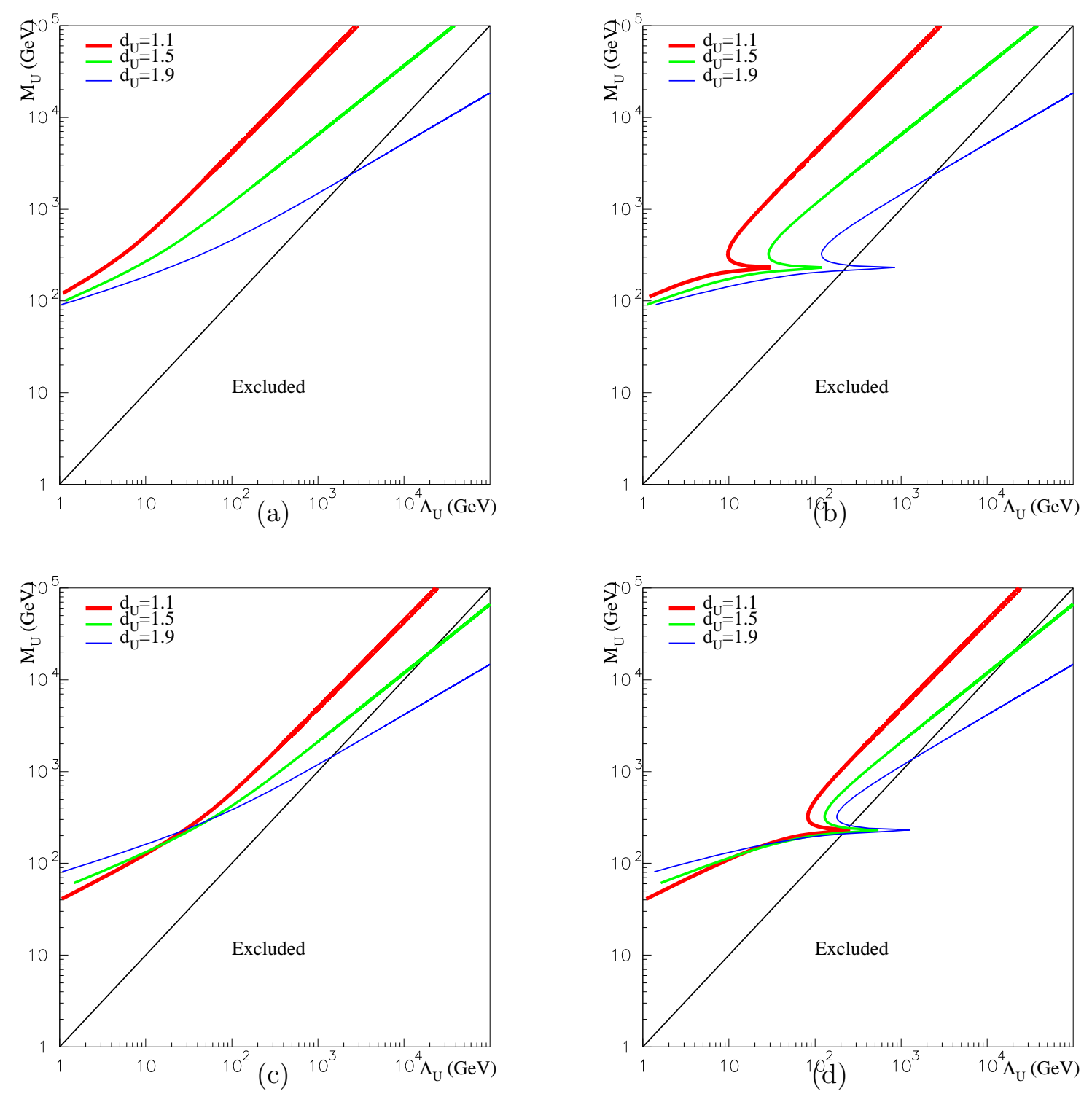

FIG. 4: The contour plot of $a_{\mu}$ with $a_{\mu}^{\mathcal{U}}=10^{-9}$ in $\Lambda_{\mathcal{U}}$ and $M_{\mathcal{U}}$ plane for $d_{\mathcal{U}}=1.1$ (red), $d_{\mathcal{U}}=1.5$ (green), and $d_{\mathcal{U}}=1.9$ (blue) with $\mu=1 \mathrm{GeV}$ in (a), (b) and $\mu=m_{Z}$ in (c), (d). $-\lambda_{f}=\lambda_{b}=-\lambda_{w}=1$ in case (a) and (c), $-\lambda_{f}=\lambda_{b}=\lambda_{w}=1$ in case (b) and (d). In the region above the corresponding curve for different $d_{\mathcal{U}}, a_{\mu}^{\mathcal{U}}$ is smaller than $10^{-9}$.

[4] K. Cheung, W. Y. Keung and T. C. Yuan, arXiv:0704.2588 [hep-ph].

[5] M. Luo and G. Zhu, arXiv:0704.3532 [hep-ph].

[6] C. H. Chen and C. Q. Geng, arXiv:0705.0689 [hep-ph].

[7] G. J. Ding and M. L. Yan, arXiv:0705.0794 [hep-ph].

[8] Y. Liao, arXiv:0705.0837 [hep-ph].

[9] T. M. Aliev, A. S. Cornell and N. Gaur, arXiv:0705.1326 [hep-ph].

[10] X. Q. Li and Z. T. Wei, arXiv:0705.1821 [hep-ph].

[11] M. Duraisamy, arXiv:0705.2622 [hep-ph].

[12] C. D. Lu, W. Wang and Y. M. Wang, arXiv:0705.2909 [hep-ph]. 
[13] M. A. Stephanov, arXiv:0705.3049 [hep-ph].

[14] P. J. Fox, A. Rajaraman and Y. Shirman, arXiv:0705.3092 [hep-ph].

[15] N. Greiner, arXiv:0705.3518 [hep-ph].

[16] H. Davoudiasl, arXiv:0705.3636 [hep-ph].

[17] D. Choudhury, D. K. Ghosh and Mamta, arXiv:0705.3637 [hep-ph].

[18] S. L. Chen and X. G. He, arXiv:0705.3946 [hep-ph].

[19] T. M. Aliev, A. S. Cornell and N. Gaur, arXiv:0705.4542 [hep-ph].

[20] P. Mathews and V. Ravindran, arXiv:0705.4599 [hep-ph].

[21] S. Zhou, arXiv:0706.0302 [hep-ph].

[22] G. J. Ding and M. L. Yan, arXiv:0706.0325 [hep-ph].

[23] C. H. Chen and C. Q. Geng, arXiv:0706.0850 [hep-ph].

[24] Y. Liao and J. Y. Liu, arXiv:0706.1284 [hep-ph].

[25] M. Bander, J. L. Feng, A. Rajaraman and Y. Shirman, arXiv:0706.2677 [hep-ph].

[26] T. G. Rizzo, arXiv:0706.3025 [hep-ph].

[27] K. Cheung, W. Y. Keung and T. C. Yuan, arXiv:0706.3155 [hep-ph].

[28] H. Goldberg and P. Nath, arXiv:0706.3898 [hep-ph].

[29] S. L. Chen, X. G. He and H. C. Tsai, arXiv:0707.0187 [hep-ph].

[30] R. Zwicky, arXiv:0707.0677 [hep-ph].

[31] T. Kikuchi and N. Okada, arXiv:0707.0893 [hep-ph].

[32] R. Mohanta and A. K. Giri, arXiv:0707.1234 [hep-ph].

[33] C. S. Huang and X. H. Wu, arXiv:0707.1268 [hep-ph].

[34] N. V. Krasnikov, arXiv:0707.1419 [hep-ph].

[35] A. Lenz, arXiv:0707.1535 [hep-ph].

[36] J. J. van der Bij and S. Dilcher, arXiv:0707.1817 [hep-ph].

[37] D. Choudhury and D. K. Ghosh, arXiv:0707.2074 [hep-ph].

[38] H. Zhang, C. S. Li and Z. Li, arXiv:0707.2132 [hep-ph].

[39] X. Q. Li, Y. Liu and Z. T. Wei, arXiv:0707.2285 [hep-ph].

[40] Y. Nakayama, arXiv:0707.2451 [hep-ph].

[41] N. G. Deshpande, X. G. He and J. Jiang, arXiv:0707.2959 [hep-ph].

[42] T. A. Ryttov and F. Sannino, arXiv:0707.3166 [hep-th].

[43] R. Mohanta and A. K. Giri, arXiv:0707.3308 [hep-ph].

[44] A. Delgado, J. R. Espinosa and M. Quiros, arXiv:0707.4309 [hep-ph].

[45] G. Cacciapaglia, G. Marandella and J. Terning, arXiv:0708.0005 [hep-ph].

[46] M. Neubert, arXiv:0708.0036 [hep-ph].

[47] M. x. Luo, W. Wu and G. h. Zhu, arXiv:0708.0671 [hep-ph].

[48] S. Hannestad, G. Raffelt and Y. Y. Y. Wong, arXiv:0708.1404 [hep-ph].

[49] N. G. Deshpande, S. D. H. Hsu and J. Jiang, arXiv:0708.2735 [hep-ph].

[50] P. K. Das, arXiv:0708.2812 [hep-ph].

[51] G. Bhattacharyya, D. Choudhury and D. K. Ghosh, arXiv:0708.2835 [hep-ph].

[52] Y. Liao, arXiv:0708.3327 [hep-ph].

[53] D. Majumdar, arXiv:0708.3485 [hep-ph].

[54] A. T. Alan and N. K. Pak, arXiv:0708.3802 [hep-ph].

[55] A. Freitas and D. Wyler, arXiv:0708.4339 [hep-ph].

[56] I. Gogoladze, N. Okada and Q. Shafi, arXiv:0708.4405 [hep-ph].

[57] C. H. Chen and C. Q. Geng, arXiv:0709.0235 [hep-ph].

[58] P. Van Nieuwenhuizen, Nucl. Phys. B 60, 478 (1973). 
[59] W. M. Yao et al. [Particle Data Group], J. Phys. G 33 (2006) 1.

[60] J. P. Miller, E. de Rafael and B. L. Roberts, Rept. Prog. Phys. 70, 795 (2007) arXiv:hep-ph/0703049.

[61] A. Datta, K. Whisnant, B. L. Young and X. Zhang, Phys. Rev. D 57, 364 (1998) arXiv:hep-ph/9705410]. 